\title{
LOCATING OSCILLATORY ORBITS OF THE PARAMETRICALLY-EXCITED PENDULUM
}

\author{
M. J. CLIFFORD and S. R. BISHOP ${ }^{1}$
}

(Received 21 December 1993; revised 8 June 1994)

\begin{abstract}
A method is considered for locating oscillating, nonrotating solutions for the parametricallyexcited pendulum by inferring that a particular horseshoe exists in the stable and unstable manifolds of the local saddles. In particular, odd-periodic solutions are determined which are difficult to locate by alternative numerical techniques. A pseudo-Anosov braid is also located which implies the existence of a countable infinity of periodic orbits without the horseshoe assumption being necessary.
\end{abstract}

\section{Introduction}

The parametrically-excited pendulum is an example of a simple one degree of freedom nonlinear system which can exhibit a plethora of nonlinear phenomena. The equation of motion is given by

$$
\ddot{\theta}+c \dot{\theta}+(1+p \cos \omega t) \sin \theta=0
$$

in which $\theta$ is the angle of rotation, $c$ is a damping constant taken as 0.1 throughout, $p$ is the scaled parametric excitation amplitude, $\omega$ is the scaled frequency of excitation and a dot represents differentiation with respect to the scaled time, $t$. Equilibria, oscillatory and rotating orbits exist and the latter two cases possess multiple attractors and can undergo symmetry-breaking and period-doubling bifurcations, with the possibility of chaotic motion [19]. Much of the bifurcational behaviour has been determined by Bryant and Miles [5] in an earlier rigorous study. Despite its simplicity, the parametrically-excited pendulum is used as a model for many physical [22] and electrical [23] systems. Mawhin [17] notes the importance of the simple pendulum in the development of modern mathematics in terms of nonlinear functional analysis and critical-point theory. Where the pendulum is used in engineering to model physical

\footnotetext{
${ }^{1}$ Centre for Nonlin. Dyn. and its Appl'ns, University College London, WC1E 6BT, U.K.

(C) Australian Mathematical Society, 1996, Serial-fee code 0334-2700/95
} 
systems, often it is necessary only to consider the oscillatory, nonrotating motions, and these will be exclusively covered in the following. It was noted with interest that an earlier study of the parametrically-excited pendulum [5] failed to locate any nonrotating solutions with odd period (except the trivial $\theta=0, \theta= \pm \pi$ solutions). This was a surprising result since there were large regions of odd periodic motions for two other pendulum systems considered [3,4]. Odd periodic solutions do exist for the parametrically-excited pendulum, but are only stable over a narrow parameter range. To locate these solutions it is necessary to use a more sophisticated approach than the usual cell-mapping or path-following techniques used in computational dynamical systems analysis.

\section{Bifurcational behaviour}

Before presenting the results in more detail, an overview of the bifurcational behaviour can be obtained by considering the bifurcation diagram of Figure 1 in the $(\omega, p)$ parameter space, which was produced by solving (1) together with cellmapping [13], path-following and bifurcation-following techniques [21], [9].

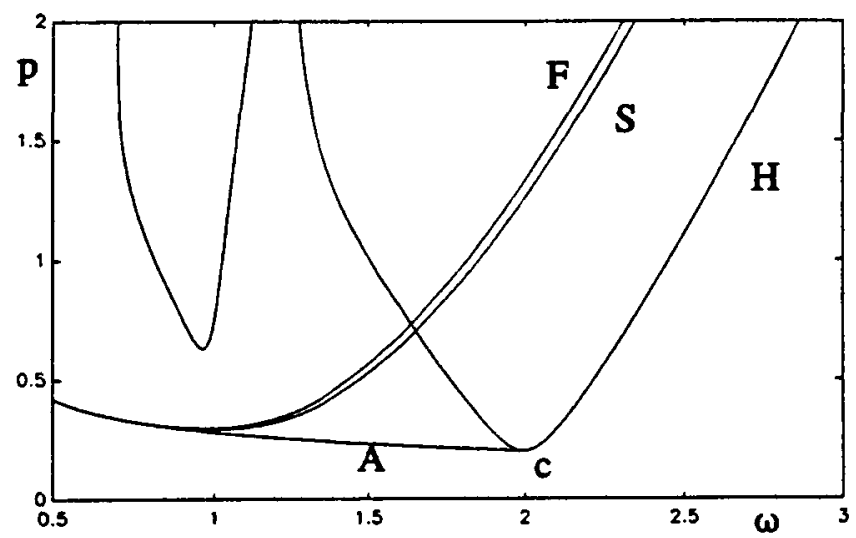

FIGURE 1. Major features of the bifurcation diagram of the parametrically-excited pendulum in the $(\omega, p)$ parameter space. $\mathrm{H}$ is where the equilibrium loses stability at a period-doubling or flip bifurcation which is subcritical to the left of $c$. $S$ is a symmetry-breaking bifurcation, and $F$ represents the end of a period-doubling cascade although only the first period-doubling is shown. The period two fold line A associated with the subcritical flip is also shown. A similar unstable zone is shown centred around $\omega=1$.

Only the major bifurcations are displayed around the primary unstable zone centred at $\omega=2$ (similar detail can be examined near other unstable zones). The line labelled $\mathrm{H}$ is where small perturbations from the equilibrium $\theta=0$ will first result in an 
initial growth which may be predicted by the linear theory $[14,12]$. To the right of the point $c$, the equilibrium bifurcates into a stable symmetric period- 2 solution at a supercritical bifurcation, whilst to the left, the resultant symmetric period- 2 solution becomes unstable at a subcritical bifurcation. Line A corresponds to a period- 2 fold where the unstable period-2 solution from the subcritical bifurcation stabilises at a saddle-node bifurcation. Line $S$ corresponds to a symmetry breaking bifurcation, where the symmetric period- 2 splits into two stable antisymmetric period-2 solutions, and line $F$ represents the end of a pair of period-doubling cascades [8], although only the first period-doubling is shown, since the cascade is very rapid. The resulting possibly chaotic attractor is stable over a very narrow parameter regime before a catastrophic bifurcation which leaves no stable oscillatory, nonrotating periodic or chaotic solutions.

The system can display complex dynamics due to the presence of homoclinic and heteroclinic tangencies of the saddles located at $\theta= \pm \pi$ (the so-called hilltop saddles) which can be accurately predicted using a Melnikov approach [15]. The existence of homoclinic and heteroclinic intersections of the saddles implies the existence of horseshoes, which in turn implies the existence of an infinite number of unstable periodic orbits [24]. The existence of unstable periodic orbits significantly affects the underlying dynamics of the system. Unstable periodic orbits can be located in the phase space by using a Newton-Raphson procedure, but the approach is considerably improved if the number of expected (unstable) periodic orbits and their approximate location is known. This information can be obtained by considering the Smale horseshoe formed by the invariant manifolds of the two hilltop saddles. The horseshoe is formed by the intersection of two ' $S$ ' shapes formed by the two stable and unstable manifolds. This is shown in figure $2 \mathrm{~A}, \mathrm{~B}$ respectively, and their intersection in Figure $3 \mathrm{~A}$. Figure $3 \mathrm{~B}$ is an idealisation of the manifolds in Figure $3 \mathrm{~A}$ and is topologically equivalent. The horseshoe formed here is similar in nature to the classic Smale horseshoe, but has three stripes and so might be termed a 3-shoe. We note that a similar horseshoe was located for the pendulum with applied torque [11]. The resulting trellis of invariant manifolds allows the required unstable periodic orbits to be located using symbolic dynamics [10].

\section{Symbolic dynamics}

The 3-shoe in Figure 4 has horizontal and vertical strips labelled $\mathrm{H} 0, \mathrm{H} 1, \mathrm{H} 2$, and $\mathrm{V} 0, \mathrm{~V} 1, \mathrm{~V} 2$ respectively such that $\mathrm{Vi}=\mathrm{G}(\mathrm{Hi}), \mathrm{i}=0,1,2$ where $\mathrm{G}$ is the Poincare map associated with the flow [25].

Each point in the horseshoe invariant set is then described uniquely by a bi-infinite symbol sequence or itinerary describing the strips visited in subsequent mappings 


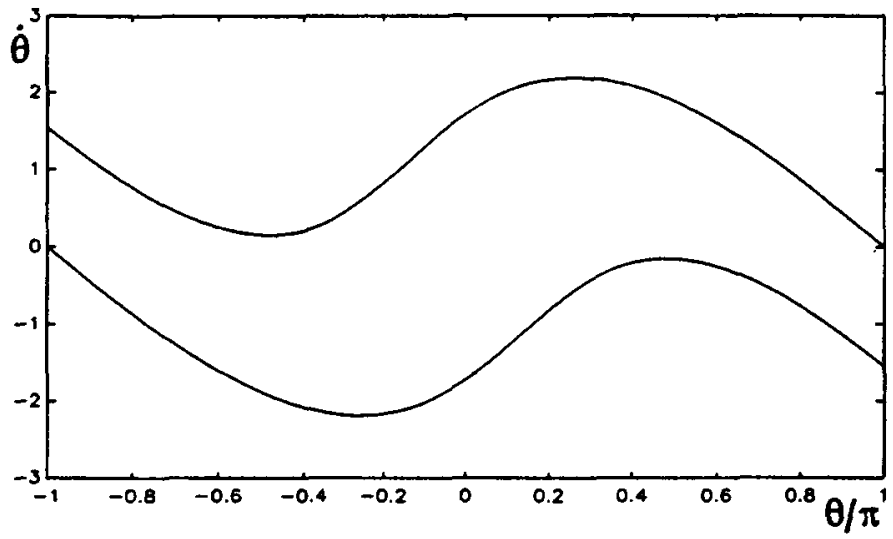

FIGURE 2A. Stable manifolds of the hilltop saddles at $\theta= \pm \pi$ with $\omega=2, p=1.8$.

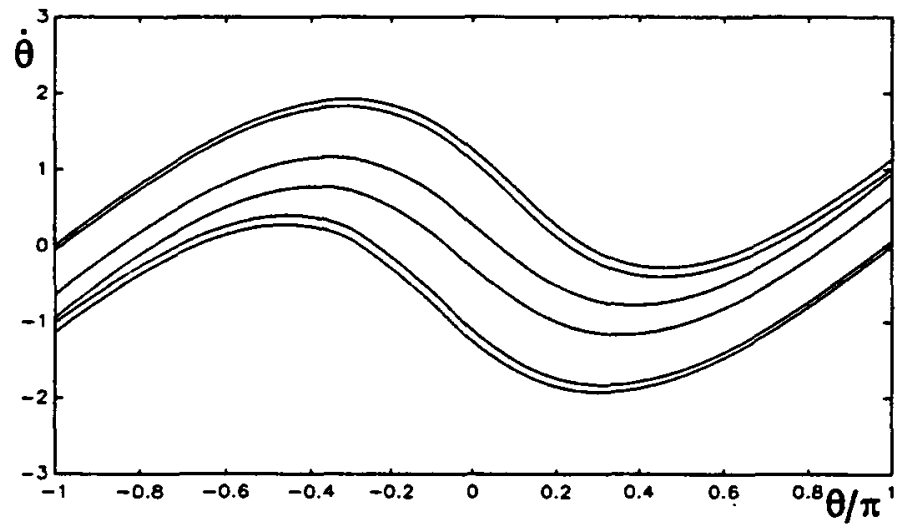

FIGURE 2B. Unstable manifolds of the hilltop saddles at $\theta= \pm \pi$ with $\omega=2, p=1.8$.

forward, and backwards in time:

$$
\ldots h_{-3} h_{-2} h_{-1} \cdot h_{0} h_{1} h_{2} h_{3} \ldots, \quad \text { where } \mathrm{G}^{\mathrm{k}}(\mathrm{x}) \in \mathrm{Hi} \Leftrightarrow h_{k}=\mathrm{i},
$$

which may be expressed identically as

$$
\ldots v_{2} v_{1} v_{0} \cdot h_{0} h_{1} h_{2} h_{3} \ldots, \quad \text { where } \mathrm{G}^{-\mathrm{k}}(\mathrm{x}) \in \mathrm{Vi} \Leftrightarrow v_{k}=\mathrm{i} .
$$

Here we concentrate on periodic orbits, which have recurrent substrings, for example ...010101.01010101 ... is one Poincaré point of a period-2 orbit, which can be identified by its 'root' $\underline{01}$. The number of periodic orbits of any particular period can be calculated, and have been tabulated for orbits up to period- 8 by McRobie [18]. 


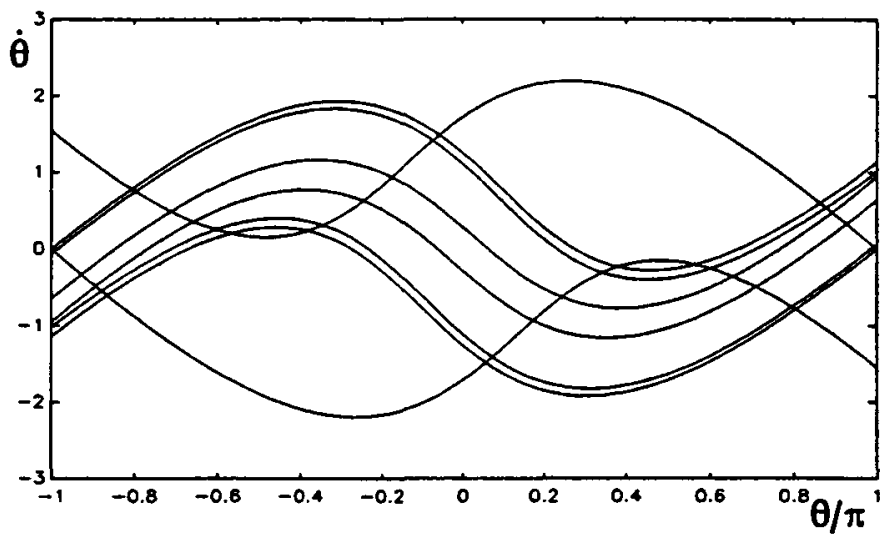

FIGURE 3A. Intersection of stable and unstable manifolds shown in Figures 2A and 2B.

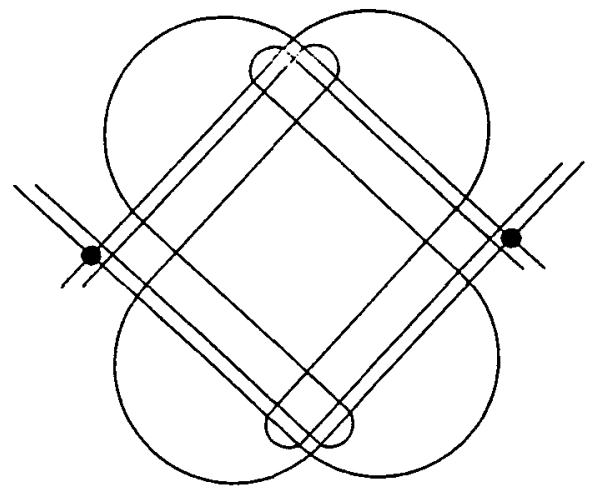

FIGURE 3B. Idealised invariant manifolds of Figure 3A, preserving the topological structure.

The itinerary can then be used to locate periodic orbits in the 3-shoe by converting the itinerary into invariant coordinates $[10]$ which uniquely describe the position of the orbit. The position of an orbit in the idealised 3-shoe can then be related to the real trellis of invariant manifolds of the saddles, giving an approximate location for the orbit in the phase space. This was carried out for all orbits up to period- 3 for the parametrically-excited pendulum, and all predicted orbits were subsequently located by using a Newton-Raphson procedure which searched for unstable periodic orbits around the approximate location given by the invariant coordinates. The location of the period-3 orbit 001 in the idealised 3-shoe and the trellis of invariant manifolds is shown in Figure 5. 


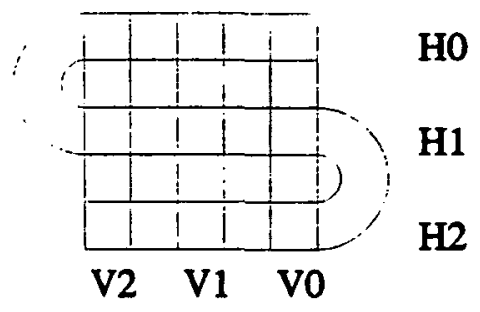

FIGURE 4. Three striped horseshoe or 3-shoe. Horizontal and vertical strips are labelled $\mathrm{Hi}$ and $\mathrm{Vi}$ respectively with $i=0,1,2$ such that $\mathrm{Vi}=\mathrm{G}(\mathrm{Hi})$ where $\mathrm{G}$ is the Poincare map associated with the flow.

Path-following these unstable orbits enables subsequent bifurcations to be identified, and in particular, stable orbits to be located. Typically the higher periodic orbits are stable only over a small parameter range, but one important result to note is that stable periodic orbits were located in the region above the chaotic zone (in terms of increasing the parametric forcing parameter, $p$ ) previously thought to contain no stable nonrotating periodic or chaotic orbits. For example, a pair of period-3 orbits are stable for $\omega=2$, and $1.6741286<p<1.6741420$. One of these orbits is the orbit 001 located earlier. The stable period-3 orbits are created at saddle node bifurcations, and rapidly undergo period-doubling bifurcations to chaotic attractors which then disappear at further bifurcations, sometimes called catastrophes.

A cell mapping program based on Hsu's method [13] with $200 \times 200$ cells in the window $\theta=-\pi$ to $+\pi, \dot{\theta}=-4$ to +4 failed to locate these stable periodic orbits since their basins of attraction are very small, and the attractors are stable over very small parameter ranges. A time history of angular displacement, $\theta$ and corresponding phase portrait in the phase space of $(\theta, \dot{\theta})$ for the stable period-3 solution, $\underline{001}$ with eigenvalues $-0.0826 \pm 0.6187 \mathrm{i}$ (stable) are shown in Figure 6.

Beside locating period-3 solutions, we can use the symbolic-dynamics approach to find higher-order periodic solutions. However, as we move to higher periods, locating solutions becomes more difficult as the orbits become increasingly unstable. Rather than trying to locate all the periodic orbits in the 3-shoe of higher period, we use a technique based on braid theory to prove the existence of many periodic solutions from the discovery of a single orbit. 

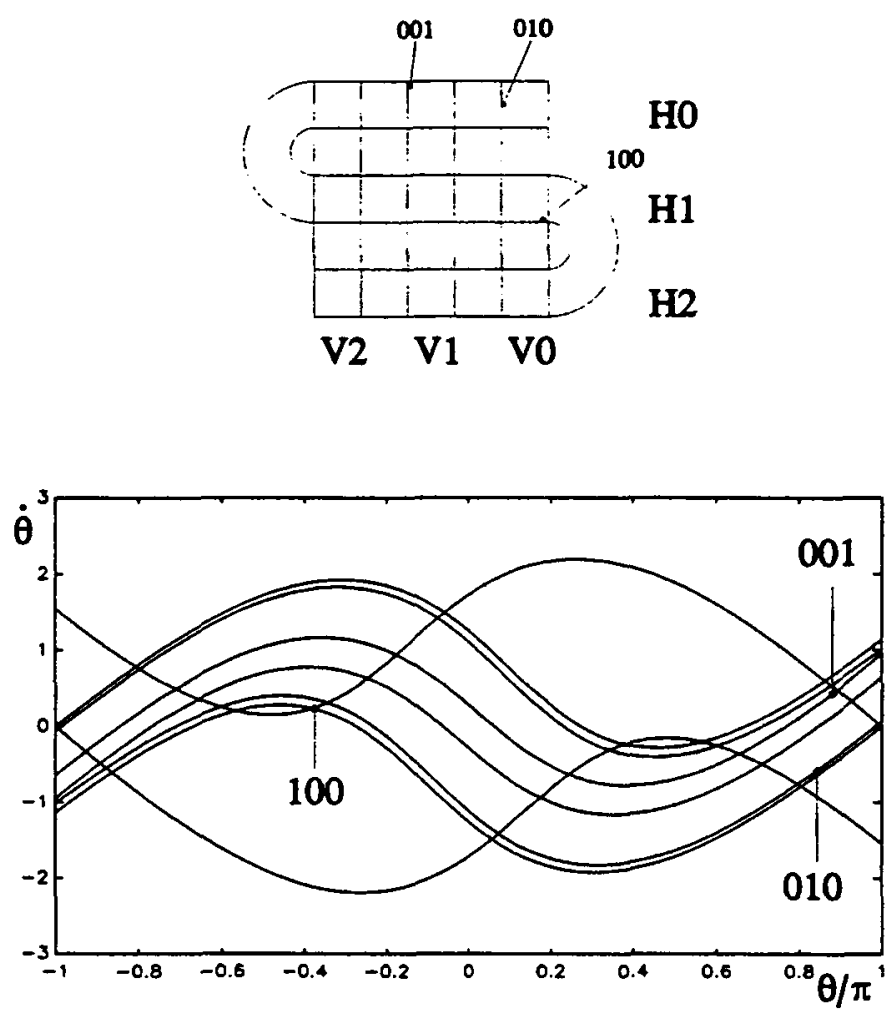

FIGURE 5. Locating the period-3 unstable orbit $\underline{001}$ in the 3-shoe and subsequently on the Poincare plane by the method of symbolic dynamics

\section{Braid diagrams}

A braid on $n$ strands may be formed from a periodic orbit with period- $n$ by plotting displacement (or angle) versus time modulo $\mathrm{T}$, where $\mathrm{T}$ is the period of the applied forcing [1]. For 3-dimensional dynamical systems all crossings of strands will be in the same sense since the strand with the lower value of $\theta(t)$ before the crossing corresponds to a higher value of the derivative $\dot{\theta}(t)$ which is perpendicular to the plane and so passes over the other. Hence all braids obtained in this manner will contain only crossings that are left over right, forming a positive braid. Braids can be classified into three classes: finite order, reducible, and pseudo-Anosov [2]. The first two classes imply little about the global dynamics of the system, whilst pseudo-Anosov braids have positive topological entropy and imply a countable infinity of (unstable) periodic orbits. 'A pseudo-Anosov braid implies chaos' [19] is the 2-dimensional analogy 

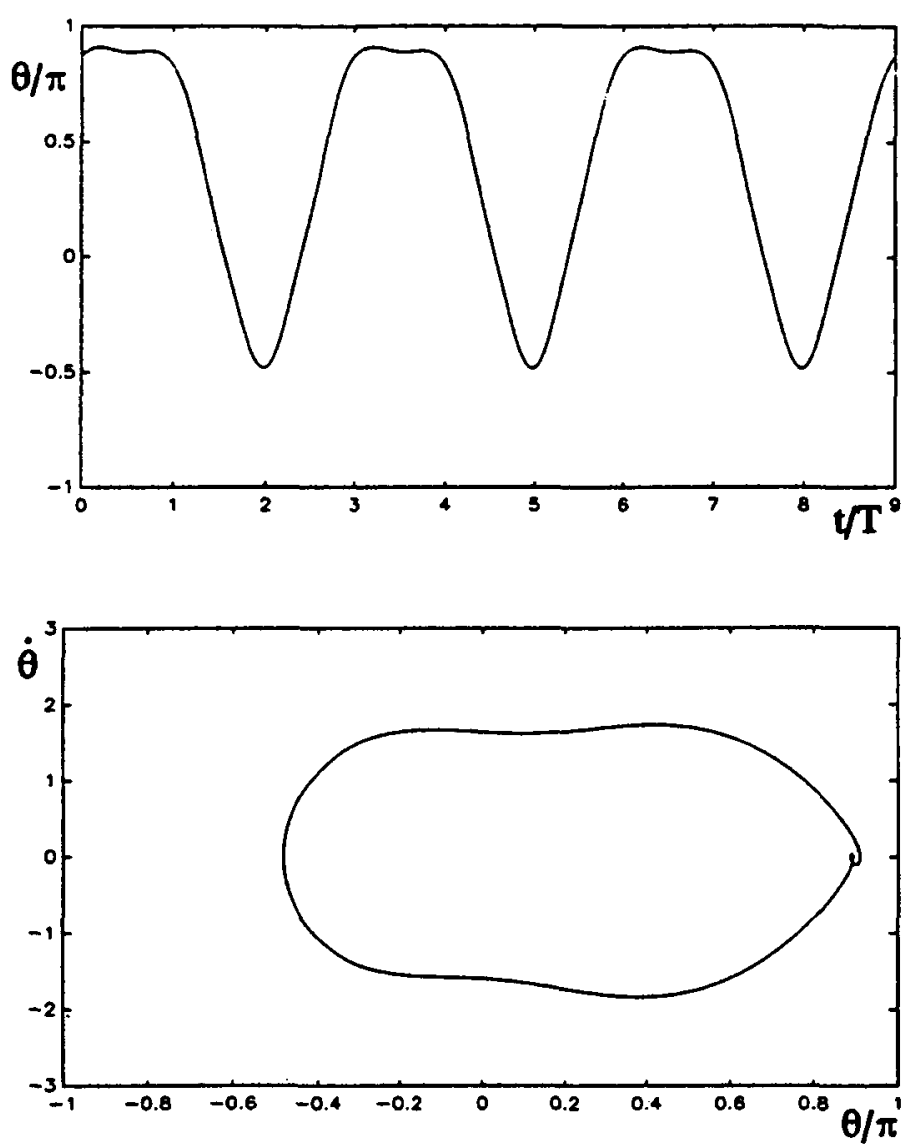

FIGURE 6. Time history and phase portrait for stable period-3 solution $\underline{001} \omega=2, p=1.67413$.

of the famous 'Period three implies chaos' statement for 1-dimensional maps [16]. None of the period-3 solutions produce pseudo-Anosov braids; indeed we have to go to period- 5 before any such solutions are located. A period-5 pseudo-Anosov braid located by the symbolic dynamics method outlined above is shown in Figure 7.

The number of orbits with a particular period that are implied by the existence of this braid can be calculated relatively easily [2], and is given in Table 1 . Thus by locating one period- 5 orbit we have proved the existence of 23 other orbits with period $\leq 10$.

This proof is independent of the 3-shoe hypothesis, and additionally we have shown that the system has positive topological entropy, that is, there exists a chaotic solution, 


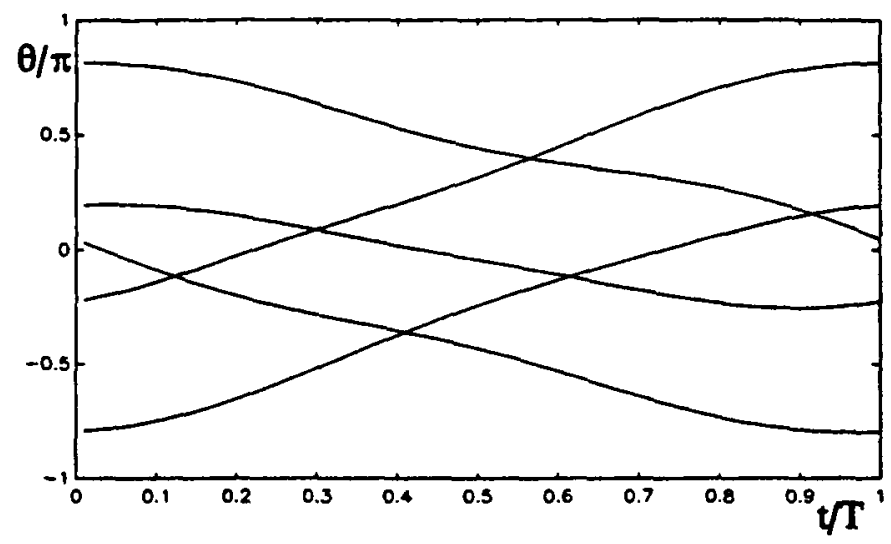

FIGURE 7. Time history of period-5 pseudo-Anosov braid for the parametrically-excited pendulum.

albeit an unstable one. This form of analysis allows a complete topological picture of the dynamics of the parametrically-excited pendulum to be built, and a partial ordering of the creation process of the nonrotating periodic orbits can also be constructed, but is beyond the scope of this paper.

\section{Conclusions}

We have highlighted the location of odd oscillatory periodic solutions for the parametrically-excited pendulum using a knowledge of the horseshoe structure formed by the invariant manifolds of two saddles. Additionally, stable periodic orbits have been located where it was initially thought that none existed for the parametricallyexcited pendulum. Basins of attraction are small and therefore in a typically 'noisy' engineering environment these would probably not be sustainable while in other more precise situations they could play a more significant role. If fine precision is available, these techniques may be used in conjunction with concepts based on control strategies to target desirable solutions [20]. In addition, we have located a pseudo-Anosov braid which implies the existence of a countable infinity of periodic orbits, positive topological entropy, and an unstable chaotic solution. These results represent a modest start towards a complete topological picture, but allow a large body of existing theory to be applied to understanding the global dynamics of the parametrically-excited pendulum. However, these results may not be extended to higher-dimensional systems since the braid theory used here is only valid in three dimensions. 
TABLE 1. Number of periodic orbits implied by period 5 pseudo-Anosov braid.

\begin{tabular}{|c|c|}
\hline Period & Number of Orbits \\
\hline 1 & 1 \\
\hline 2 & 1 \\
\hline 3 & 2 \\
\hline 4 & 1 \\
\hline 5 & 3 \\
\hline 6 & 2 \\
\hline 7 & 4 \\
\hline 8 & 3 \\
\hline 9 & 4 \\
\hline 10 & 2 \\
\hline
\end{tabular}

\section{Acknowledgements}

The authors would like to acknowledge the helpful comments and contributions of Allan McRobie and the referee, and the financial support of the Science and Engineering Research Council.

\section{References}

[1] J. S. Birman, Braids, links, and linking class groups, Annals of Math. Study 82 (Princeton University Press, 1974).

[2] P. L. Boyland and J. Franks, Notes on dynamics of surface homomorphisms (Mathematics Institute, Warwick University, 1989).

[3] P. J. Bryant and J. W. Miles, "On a periodically forced, weakly damped pendulum. Part 1: Applied torque”, J. Austral. Math. Soc. Ser. B 32 (1990) 1-22.

[4] P. J. Bryant and J. W. Miles, "On a periodically forced, weakly damped pendulum. Part 2: Horizontal forcing", J. Austral. Math. Soc. Ser. B 32 (1990) $23-41$.

[5] P. J. Bryant and J. W. Miles, "On a periodically forced, weakly damped pendulum. Part 3: Vertical forcing”, J. Austral. Math. Soc. Ser. B 32 (1990) 42-60.

[6] D. Capecchi and S. R. Bishop, "Periodic oscillations and attracting basins for a parametrically excited pendulum", Dynamics and Stability of Systems, 9 (1994) 123-143.

[7] M. J. Clifford and S. R. Bishop, "Generic features of escape from a potential well under parametric excitation", Physics Letters A 184 (1993) 57-63.

[8] M. J. Feigenbaum, "Universal behaviour in nonlinear systems", Los Alamos Science 1 (1980) 4-27.

[9] S. Foale and J. M. T. Thompson, "Geometrical concepts and computational techniques of nonlinear dynamics", Computer methods in Applied Mechanics and Engineering 89 (1991) 381-394. 
[10] J. Guckenheimer and P. Holmes, Nonlinear oscillations, dynamical systems, and bifurcations of vector fields (Springer-Verlag, New York, 1983).

[11] E. G. Gwinn and R. M. Westervelt, "Horseshoes in the driven, damped pendulum", Physica D 23 (1986) 396-401.

[12] C. Hayashi, Nonlinear oscillations in physical systems (McGraw-Hill, New York, 1964).

[13] C. S. Hsu, Cell to cell mapping: a method of global analysis for nonlinear systems (Springer-Verlag, New York, 1987).

[14] D. W. Jordan and P. Smith, Nonlinear ordinary differential equations (Oxford University Press, New York, 1987).

[15] B. P. Koch and R. W. Leven, "Subharmonic and homoclinic bifurcations in a parametrically forced pendulum", Physica D 16 (1985) 1-13.

[16] T. Li and J. A. Yorke, "Period three implies chaos", Amer. Math. Monthly 82 (1975) 481-485.

[17] J. Mahwin, "The forced pendulum: a paradigm for nonlinear analysis and dynamical systems", Expositiones Mathematicae 6 (1988) 271-287.

[18] F. A. McRobie, "Bifurcational precedences in the braids of periodic orbits of spiral 3-shoes in driven oscillators", Proc. R. Soc. Lond. A 438 (1992) 545-568.

[19] F. A. McRobie and J. M. T. Thompson, "An introduction to braids and knots in driven oscillators", Int. J. Bifurcation and Chaos, 3 (1993) 1343-1361.

[20] E. Ott, C. Grebogi and J. A. Yorke, “Controlling chaos", Phys. Rev. Lett. 64 (11) (1990) 1196-1199.

[21] T. S. Parker and L. O. Chua, Practical numerical algorithms for chaotic systems (Springer-Verlag, New York, 1989).

[22] R. C. T. Rainey, "The dynamics of tethered platforms", Trans. Royal Institute of Naval Architects 120 (1978) 59-80.

[23] F. M. Salam and S. S. Sastry, "Dynamics of the forced Josephson junction: the region of chaos", IEEE Trans. Circuits and Systems CAS-30 (1985) 784-796.

[24] S. Smale, “Differential dynamical systems", Bull. Amer. Math. Soc. 73 (1967) 747-817.

[25] J. M. T. Thompson and H. B. Stewart, Nonlinear dynamics and chaos (Wiley, Chichester, 1986). 